\title{
Resistance of melanoma cells to anticancer treatment: a role of vascular endothelial growth factor
}

\author{
Joanna Bogusławska-Duch, Magdalena Ducher, Maciej Małecki \\ Department of Applied Pharmacy, Medical University of Warsaw, Warsaw, Poland
}

\begin{abstract}
Melanoma is one of the most aggressive and resistant to treatment neoplasms. There are still many challenges despite many promising advances in anticancer treatment. Currently, the main problem for all types of treatment is associated with heterogeneity. Due to heterogeneity of cancer cells, "precise" targeting of a medicine against a single phenotype limits the efficacy of treatment and affects resistance to applied therapy. Therefore it is important to understand aetiology and reasons for heterogeneity in order to develop effective and long-lasting treatment. This review summarises roles of vascular endothelial growth factor (VEGF) that may stimulate growth of a melanoma tumour irrespective of its proangiogenic effects, contributing to cancer heterogeneity. VEGF triggers processes associated with extracellular matrix remodelling, cell migration, invasion, angiogenesis, inhibition of immune responses and favours phenotypic plasticity and epithelial-mesenchymal transition. Consequently, it participates in mechanisms of interactions between melanoma cancer cells and microenvironment and it can modify sensitivity to therapeutic factors.
\end{abstract}

Key words: cancer, melanoma, vascular endothelial growth factor, resistance to therapy, heterogeneity.

\section{Introduction}

Melanoma is a malignant tumour with an extremely aggressive character which is expressed in early metastases and resistance to applied treatment [1]. It is interesting to observe that this fact is determined by the cancer aetiology itself. Pigment cells (melanocytes) subjected to neoplastic formation originate from neural crest cells (NCCS) that during embryonic development colonise mainly areas of the skin, including the eye, and some other tissues in the body, but to a lesser extent. Therefore, melanoma cells show such properties as phenotypic plasticity or high migration potential [2-5].

Cancer cell heterogeneity with regard to a phenotype and functions can be observed for many types of cancer and it contributes to resistance $[6,7]$. Heterogeneity of melanoma cells may be a result of genetic, epigenetic and environmental changes inside a tumour as well as of the presence of cancer stem cells (CSCS) that gather in vascular niches allowing for cancer progression [7]. Interactions between cancer and the microenvironment are critical for melanoma invasion and metastases. Growth factors participating in such relations include vascular endothelial growth factor (VEGF) [8] that may stimulate cancer growth apart from its proangiogenic effects. VEGF is synthesised by many types of cells in the environment with an insufficient amount of oxygen [9]. It has been discovered that the majority of melanoma cancer cells has VEGF receptors on their surface and via autocrine or paracrine mechanisms signalling pathways are triggered causing increase in tumour proliferation and resistance to treatment $[10,11]$.

The paper presents mechanisms of interactions between melanoma and microenvironment with special attention placed on the ability of VEGF to modify sensitivity to therapeutic factors. It is fundamental to understand roles of VEGF in the development of this disease in an appropriate way in order to design innovative and effective anticancer agents with significantly improved outcomes of clinical trials.

\section{Tumour microenvironment and resistance to treatment}

Recently, treatment of cancer, including melanoma, has been revolutionised thanks to targeted treatment and immunotherapy. However, the efficacy of these approaches is limited by progressive resistance to medica-

Address for correspondence: Joanna Bogusławska-Duch MSc, PhD student, Department of Applied Pharmacy, Medical University of Warsaw, 1 Banacha St, 02-097 Warsaw, Poland, e-mail: jboguslawska1@wum.edu.pl Received: 3.01.2019, accepted: 21.02.2019. 
tions [12, 13]. A number of various mechanisms cause resistance to anticancer treatment. In case of melanoma, primary (innate) resistance depending on the biology of cancer cells before the start of treatment and acquired (secondary) resistance, which appears during therapy, are both possible $[14,15]$. Mechanisms of primary resistance in melanoma include, among others, mutations in the RAC1 or PTEN genes and mechanisms of acquired resistance are associated with BRAF or NRAS mutations [14]. Numerous studies indicate that tumour microenvironment (TME) plays a critical role in disease progression [16]. Cancer cells strictly collaborate with tumour microenvironment cells, namely stromal cells, and as a result, conditions that favour tumour development are created [17]. Melanoma stromal cells include, among others, fibroblasts, endothelial cells and immune cells [18]. They stimulate rapid growth of cancer, metastasis formation and resistance to treatment because they trigger processes associated with extracellular matrix remodelling, cell migration, invasion, angiogenesis and inhibition of immune responses (immunosuppression) as they produce cytokines, chemokines and growth factors [17].

A vascular niche is a special region in the tumour microenvironment as it is the place where cancer stem cells with resistance to the majority of applied therapies are gathered. These cells show the potential for indefinite self-renewal and asymmetric division that contributes to increased differentiation of daughter cells (including progenitor cells and differentiated cancer cells) [19]. Interestingly, thanks to phenotypic plasticity of the melanoma cell population, depending on the genotype and microenvironment, they may become undifferentiated and return to the pool of cancer stem cells [20]. A vascular niche is a separate region of a tumour where CSCs, showing phenotypic plasticity mentioned above, are protected from the immune system and at the same time increase cancer metastatic potential [19]. Moreover, a vascular niche was initially understood as a place that does not cause hypoxia thanks to a relationship between CSCs and endothelial cells. Whereas CSCs are characterised by their resistance to hypoxia $[21,22]$ and therefore they have the potential for phenotypic transformation [23].

\section{VEGF and its receptors on cancer cells}

Vascular endothelial growth factor was identified and isolated as a mitogen that is specific to endothelial cells and its role is to control processes associated with proliferation of blood and lymphatic vessels and with permeability of their walls [24-26]. VEGF is currently known as VEGF-A and belongs to a family of growth factors that also includes VEGF-B, VEGF-C, VEGF-D and placental growth factor (PIGF). Members of this family show various expression depending on the cell type, affinity to receptors and biological functions. VEGF (namely VEGF-A) is the most interesting one and has several different variants resulting from alternative splicing of the VEGF gene (VEGF121, VEGF145, VEGF148, VEGF165, VEGF183, VEGF189 and VEGF206) $[27,28]$. Studies have shown that isoforms predominant in melanoma include VEGF165 and VEGF121 [29].

It has been demonstrated that numerous types of cells can release VEGF and therefore functions of this growth factor are extended [30]. One of the most interesting discoveries is related to VEGF signalling in melanoma cancer cells as it indicates there are specific receptors on their surface that are mediators in this signalling. Melanoma cells express receptors for VEGF with the activity of tyrosine kinase: VEGFRs (vascular endothelial growth factor receptors) and neuropilin: NRPs (neuropilins) $[8,28]$. Classic VEGFRs include VEGFR-1 (FLT1), VEGFR-2 (FLK1/ KDR) and VEGFR-3 (FLT4) [31]. VEGF receptors consist of three domains: extracellular domain responsible for ligand binding, transmembrane domain and intracellular domain with tyrosine kinase and a C-terminal fragment, namely autophosphorylation site [32]. It should be mentioned that VEGF shows affinity to VEGFR-1 and VEGFR-2 [27]. However, it is VEGFR-2 that is a predominant receptor related to tyrosine kinase (RTK) used to trigger VEGF signalling in cells [31]. On the other hand, VEGFR-3 binds to VEGF-C and VEGF-D, and in consequence it regulates lymphangiogenesis [27, 32]. Moreover, soluble VEGFR-1 shows extremely high affinity to VEGF and it may play a role of its natural antagonist [33]. Although these receptors were initially thought to be active only in endothelial cells, now it is known that the majority of them is expressed in numerous types of cancer cells [28]. The majority of melanoma cells show a high expression of VEGFR-1, VEGFR-2 and VEGFR-3, which correlates with cancer clinical features [11, 34, 35]. Cancer cells that do not express one or more VEGFRs are supported by neuropilins. Neuropilin-1 (NRP-1) and neuropilin-2 (NRP-2) belong to transmembrane proteins and their amino acid sequences are homologous in 44\% [36, 37]. They consist of an extracellular segment, transmembrane segment and a short cytoplasmic segment which does not exhibit any tyrosine kinase activity [38]. Neuropilins are co-receptors that enhance a response to growth factors and other mediators in physiological and pathological conditions. They, among others, form complexes with VEGFRs and increase their affinity to proteins from the VEGF family [36, 37]. NRP-1 found in melanoma cells acts together with VEGF and VEGFR-2 [39], whereas NRP-2 binds to VEGF-C and supports the activity of VEGFR-3 in cancer development $[40,41]$. Furthermore, NRP-1 plays a role in the survival and self-renewal of melanoma stem cells [42].

\section{Functions of VEGF in melanoma tumour}

Vascular endothelial growth factor secreted by melanoma cells and stromal cells (including endothelial cells, macrophages and fibroblasts) has numerous functions in 
the tumour microenvironment. They are connected with the aforementioned capacity to interact with VEGFRs and NRPs leading to the activation of numerous signalling pathways in tumour cells that are responsible for cancer progression, metastasis formation and resistance to therapy [8, 43-45].

VEGF is first of all a proangiogenic factor playing a key role in the angiogenesis process [46]. The most potent stimulus contributing to cancer angiogenesis includes hypoxia as it leads to the activation of hypoxia-inducible factor 1 (HIF-1). HIF-1 consists of the HIF-1 $\alpha$ subunit sensitive to the oxygen levels, and HIF-1 $\beta$ subunit that is subject to constitutive expression. It is worth mentioning that there are mechanisms independent of hypoxia, which lead to stabilisation of HIF-1 $\alpha$ in melanoma and in consequence increase the possibility of VEGF synthesis [47]. Such proangiogenic activators include BRAFV600E mutation, phosphoinositide 3-kinase (PI3K), endotelin-1, microphthalmia-associated transcription factor (MITF) and nuclear factor $\kappa \mathrm{B}(\mathrm{NF}-\kappa \mathrm{B})$, NRAS mutation with GAB2 protein or reactive oxygen species (ROS) [47]. Studies show that autocrine VEGF signalling in melanoma cells via VEGF specific receptors enables growth, survival, migration and invasion of cancer cells [8, 28]. Additionally, VEGF plays a role in promoting CSCS as it regulates the vascular niche and innate properties of cancer stem cells [42], thus creating favourable conditions for phenotypic plasticity and epithelial-mesenchymal transition (EMT) [48] (Figure 1).

Moreover, this factor may participate in the formation of melanoma vasculogenic mimicry, which is responsible for a malignant phenotype of this cancer and the patient's poor prognosis [49]. Interestingly, cancer cells able to generate vasculogenic mimicry are associated with CSCS and EMT [49-51]. VEGF also plays a key role in the suppression of immune responses targeted against melanoma [52] and activation of anti-apoptotic proteins [8]. The aforementioned VEGF functions are of great significance in mechanisms of resistance of melanoma to administered medications.

\section{Mechanisms of melanoma resistance}

Understanding of molecular pathways responsible for resistance makes it possible to anticipate a probable cancer response to applied treatment and to better prepare for such a response. Melanoma resistance is caused by mechanisms that are triggered inside cancer cells as well as changes inside stem cells and tumour microenvironment (stromal cells/microenvironment stroma). These mechanisms may act independently or together and via various signalling pathways. Such differences are a result of differences among tumours and therefore it is necessary to adopt an individual approach to treatment $[53,54]$.

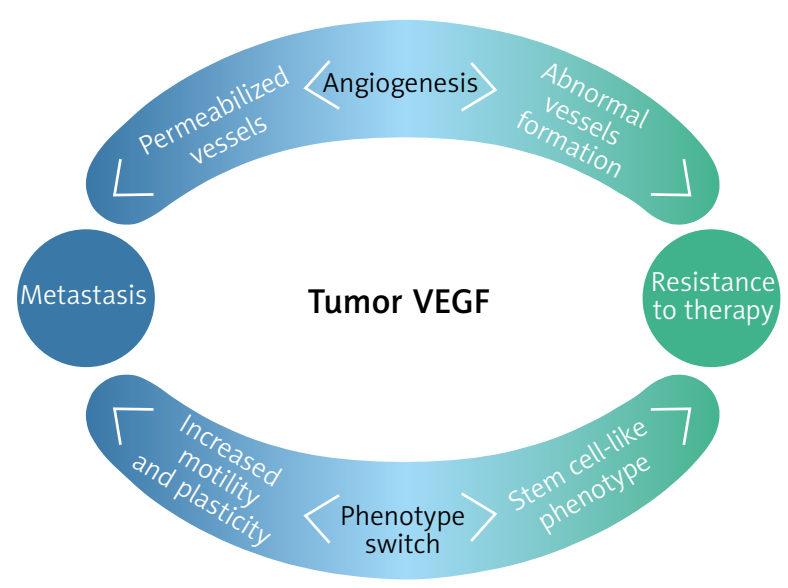

Figure 1. Multiple roles of VEGF in melanoma progression

Below are presented mechanisms of melanoma resistance, taking into account examples of the effect of proangiogenic VEGF on particular mechanisms.

\section{$A B C$ transporters as protein efflux pumps for drugs}

ATP binding transporters (binding cassette transporters; $A B C$ ) are multi-domain transmembrane proteins that use energy from ATP hydrolysis to pump substances through the cell membrane $[55,56]$. Basic structural elements of the $A B C$ protein are as follows: nucleotide binding domains (NBDs) and transmembrane domains (TMDs). TMDs are responsible for specificity of transported substrates and NBDs for ATP binding and hydrolysis [57]. A phenomenon of resistance to anticancer agents is considered to be the main function of $A B C$ transporters in progression of oncological diseases. These proteins inhibit effective therapy as they remove medications from cancer cells. $A B C$ melanoma proteins that have been identified include: ABCA9, ABCB1, ABCB5, ABCB8, ABCC2, $A B C D 1$ and $A B C G 2[57,58]$.

Akiyama et al. described this mechanism of resistance using the $A B C B 1$ protein (i.e. glycoprotein $P$ ) encoded by the MDR1 gene (multidrug resistance gene 1) and cooperation between melanoma cells and stromal cells. The study showed that tumour endothelial cells (TECS) acquire chemoresistance to paclitaxel by increasing expression of MDR1 due to the activity of VEGF from cancer cells [59].

Another $A B C$ transporter playing a vital role in malignant skin cancer progression is ABCB5 that is used to identify melanoma stem cells (MSCS). MSCs, called also malignant melanoma-initiating cells (MMICs), belong to the aforementioned subpopulation of malignant cells that are capable of self-renewal and as a consequence lead to cancer development $[60,61]$. Recent studies show 
that ABCB5 is not only a MSC marker. It plays an essential role in relations between maintenance of MMICs, drug resistance and melanoma tumour growth [61]. Chartrain et al. confirmed that melanoma cells with ABCB5 expression were resistant to numerous chemotherapeutic agents that are currently used [62]. On the other hand, Wilson et al. proved that ABCB5 (+) melanoma stem cells drive secretion of IL-1b to trigger the IL-1b/L8/CXCR1 pathway that stimulates ABCB5 (-) melanoma cells and contributes to resistance [61]. Studies conducted by Frank et al. showed that ABCB5 (+) MSCs possess VEGFR1 (vascular endothelial growth factor receptor-1) activity that controls vasculogenic mimicry and produces a direct effect on melanoma drug resistance [63].

\section{Survival and proliferation pathways}

Another strategy of cancer cells used to inhibit the efficacy of treatment is enhanced expression of pathways involved in cancer proliferation and survival. With regard to melanoma RAS-RAF-MEK-ERK (MAPK, mitogenactivated protein kinase) and PI3K-AKT signalling pathways can be differentiated, which in addition can interact with each other $[64,65]$. They are activated, among others, by NRAS, BRAF mutations as well as by loss of PTEN properties (PI3K kinase phosphatase) and stimulation of growth factors including VEGF. This mechanism of resistance leads to increased tumour growth, survival, angiogenesis and metastasis formation [66, 67]. Using three dimensional melanoma cultures as early as in 2006 it was demonstrated that both signalling pathways (MAPK; PI3K-AKT) must be suppressed in order to inhibit cell proliferation [68]. Studies by Shannan et al. conducted on the 3D melanoma model confirmed that increase in potential of PI3K inhibitors was possible when combined with treatment suppressing the BRAF mutation [69].

One of more interesting discoveries associated with VEGF includes its ability for paracrine, autocrine and intracrine regulation associated with cancer cells and resulting in control of the above-mentioned pathways [10, 28, 70, 71].

Melanoma VEGF is mainly produced in cancer cells $[8,10]$ and it acts paracrinely on VEGFRs and NRPs of endothelial cancer cells, among others, leading to angiogenesis and permeability of the blood vessels. Another function of this factor includes autocrine stimulation of tumour growth that consists in VEGF binding to VEGFRs and NRPs of cancer cells [10]. Moreover, melanoma cells without receptors to bind VEGF promote their survival via intracellular intracrine mechanisms where ligand release from the cell is not necessary [10, 70].

Adamcic et al. demonstrated that primary and metastatic melanoma cells may trigger the VEGF/VEGFR2 signalling pathway via autocrine and intracrine mechanisms favouring tumour development through stimulation of MAPK and PI3K pathways [10]. On the other hand, Beck et al. using a murine skin cancer model showed the effects of the VEGF/VEGFR2 pathway in cancer endothelial cells on the development of vascular niches in cancer stem cells (CSCS) and cancer growth via autocrine effects of VEGF on NRP1 CSCs [42]. Additionally, Ruffini et al. found that VEGF worked with NRP1 and VEGFR2 to promote migration and invasion of melanoma cells [39]. Moreover, NRP1 expression is associated with increased invasiveness of melanoma cells, even in case of lack of VEGFR2 expression thanks to further activation of pathways with AKT [39, 72]. These signalling pathways in melanoma lead to resistance to treatment, especially with BRAF inhibitors. Recent research on the resistance of melanoma to vemurafenib demonstrates that VEGF belongs to key oncogenic factors involved in a pathological mechanism associated with the BRAF (V600E) mutation in cancer cells [73].

\section{Anti-apoptotic pathways}

Apoptosis plays a vital role in the maintenance of appropriate development and homeostasis of body tissues [74]. A characteristic of cancer cells is defectiveness of this process, which is determined by resistance to apoptotic signals. Pathologic conditions associated with avoiding death by melanoma cells are a result of adaptation to the microenvironment and applied treatment [75].

Cell apoptosis is controlled by numerous signalling pathways and the most important ones are: external (via receptors) and internal (via mitochondria) [76, 77]. The external pathway is triggered by binding appropriate ligands (TNF- $\alpha$, CD95L/FasL or TRAIL) to death receptors. Resulting complexes activate caspases leading to rapid cell death. On the other hand, the internal pathway may be activated due to stressed-out endoplasmic reticulum, DNA defects, hypoxia or metabolic stress. Death signals lead to activation of pro-apoptotic proteins belonging to the $\mathrm{Bcl}-2$ family, resulting in increased permeability of the mitochondrial membrane. As a result, the cytochrome $c$ is released into the cytoplasm which in a complex with Apaf- 1 activates caspases and also contributes to cell death [78-80]. Proteins from the Bcl-2 family mentioned above play a significant role in control of mitochondrial apoptosis. They include anti-apoptotic proteins counteracting apoptosis (i.e. Bcl-2, Bcl-XL, BCl-w, Mcl-1) and proapoptotic proteins (e.g. Bid, Bak, Bad, Bax, Noxa) [81].

High activity of MAPK and PI3K pathways specific to melanoma contributes to imbalance between proapoptotic and anti-apoptotic proteins causing increased levels of the following anti-apoptotic proteins: $\mathrm{Bcl}-2$, Mcl-1, Bcl$X \mathrm{~L}$ and livin, survivin and XIAP belonging to inhibitors of apoptosis (IAPS) $[75,77]$. VEGF is also involved in these abnormal processes and it leads not only to angiogenesis but also to the inhibition of apoptosis in tumours [8]. Iervolino et al. were the first ones to confirm, using an example of melanoma cells, that overexpression 
of Bcl-2 and hypoxia worked synergistically in order to modulate VEGF via the effects on mRNA-VEGF stabilisation and HIF-1 activity [82]. Bcl-2 may regulate the expression of HIF-1 and VEGF indirectly via stimulation of PI3K and MAPK signalling pathways [83] or directly by forming a triple complex with the HIF-1a subunit and HSP9O chaperone protein [84]. Moreover, it has been confirmed that in endothelial cells Bcl-XL can stimulate VEGF when it is activated by EGF (epidermal growth factor). This factor is secreted outside the cell and via an autocrine mechanism it binds to VEGFR2 causing expression of Bcl2. This mechanism results in increased tumour survival and development $[85,86]$. Studies conducted by Zeitlin et al. also demonstrated mechanisms linking VEGF and $\mathrm{BCl}-2$ that concerned endothelial cells and cancer cells, and a mutual correlation between them $[87,88]$.

Yan et al. identified VEGF as a regulator of livin expression in melanoma cells. The inhibition of the VEGF/ mTOR/livin pathway may be an additional strategy to inhibit viability of melanoma cancer cells [89]. Survivin is another protein from the IAP family which interacts with VEGF. Cancer cells show an increased expression of survivin that stimulates synthesis/release of VEGF via mechanisms in a manner that is dependent on b-catenin. After release, VEGF may affect endothelial cells and increase angiogenesis. Alternatively, in melanoma tumours this mechanism may lead to promotion of vasculogenic mimicry [90].

\section{Immunosuppressive cytokines and resistance to treatment}

Immunosuppression is one of mechanisms involved in resistance to therapy in the tumour microenvironment [91]. The most recognised and characterised cancer immunosuppressive cytokines include: interleukin-10 (IL-10), transforming growth factor $\beta$ (TGF- $\beta$ ) and vascular endothelial growth factor (VEGF) [92, 93]. Important mechanisms where IL-10, TGF- $\beta$ and VEGF counteract development of a cancer immune response include induction of accumulation of immature myeloid-derived suppressor cells (MDSCs) and regulatory T-cells as well as inhibition of migration of T-cells into a tumour [92, 94].

Research proves that the PTEN mutation in melanoma cells increases expression of immunosuppressive factors, especially VEGF, leading to reduced infiltration of T-cells in a tumour and thereby worse outcomes of treatment with PD-1 inhibitors [95]. Immunosuppressive agents that inhibit the PD-1 receptor or PD-L1 ligand act to inhibit inactivation of T-cells. As a result, a stronger immune response against cancer cells is activated [96]. In a murine melanoma model Shrimali et al. demonstrated that VEGF inhibition causes growth of T-cells in a tumour [97].

Moreover, VEGF overexpression in a melanoma tumour leads to dysfunction of dendritic cells associated with their immaturity to play a key role in the regulation of the immune system. As a result, cancer escapes from an immune response and the tumour grows [98].

Research by Agostino et al. demonstrated there is a correlation between VEGF expression in the serum and growth of regulatory T-cells in the peripheral blood in melanoma patients [99].

On the other hand, many studies confirm that melanoma cells with the BRAF(V600E) mutation show an increased expression of VEGF, an immunosuppressive mediator, which results in recruitment of inflammatory cells (MDSC; regulatory T-cells) mentioned earlier. Moreover, it has been demonstrated that in such conditions melanoma cells (BRAFV600E) may reduce maturity of dendritic cells and inhibit their ability to produce IL-12 and TNF- $\alpha$ [100].

Interestingly, macrophages, namely inflammatory cells that reside inside a tumour, have mainly a progressive effect on the development of cancer tissue and as a result, it is not possible to trigger an immune response targeted against cancer [101]. Furthermore, studies by Wang et al. proved that BRAF inhibitors used in melanoma activated the MAPK pathway in macrophages to produce VEGF. Release of this cytokine leads to proliferation of immunologically inactive macrophages as well as triggering of VEGF/VEGFRs pathways in melanoma cells resulting in resistance and tumour growth [44].

Mechanisms presented above prove that combined treatment is necessary to achieve therapeutic success as only such combination therapy can effectively counteract resistance.

\section{Combined treatment as a strategy to improve therapeutic outcomes}

The use of targeted treatment is a real breakthrough in the treatment of oncological patients. Unfortunately, success of targeted treatment is often suppressed by developing resistance. Understanding mechanisms of melanoma resistance is a necessary strategy in order to improve efficacy and to prolong effects of modern anticancer therapies $[13,102]$. Improvement of diagnostic methods is also a real challenge - they should not only consider genetic differences between tumours in various patients, but also genetic and phenotypic heterogeneity in the same patient and within one tumour as well. Furthermore, recent studies show that in early tumour growth, the endothelial cell population in melanoma includes endovascular progenitors (EVPS) initiating a vasculogenic process, for which anti-VEGF therapy was not sufficient [103]. This finding confirms the importance of understanding endothelial heterogeneity, which opens up new possibilities for more effective anti-vascular therapies in cancer.

There are ongoing studies on combined treatment that would successfully eliminate all types of cancer cells found in a heterogeneous tumour resulting in the 
Table 1. Currently conducted clinical trials in melanoma using an antiangiogenic agent that inhibits VEGF activity [105]

\begin{tabular}{ll}
\hline ID number & Investigated drugs \\
\hline NCT01879306 & Bevacizumab, paclitaxel, ipilimumab \\
\hline NCT02158520 & Bevacizumab, paclitaxel, ipilimumab \\
\hline NCT00790010 & Bevacizumab, ipilimumab \\
\hline NCT01950390 & Bevacizumab, ipilimumab \\
\hline NCT01984242 & Bevacizumab, atezolizumab, sunitinib \\
\hline
\end{tabular}

elimination of primary resistance and preventing development of secondary resistance. The table below demonstrates various configurations of combination therapies against melanoma in clinical trials that contain antiangiogenic drug (bevacizumab) directly inhibiting the activity of VEGF (Table 1) [104, 105]. It is worth citing the AVAST-M trial, which involved the use of Avastin (also known as Bevacizumab) in the treatment of patients at high risk of melanoma recurrence. In the study, adjuvant bevacizumab significantly improved the disease-free interval, but did not improve overall survival. The results also confirmed the hypothesis that combining bevacizumab with adjuvant immune checkpoint inhibitors is beneficial for melanoma patients with the high-risk BRAF mutation [106].

\section{Acknowledgments}

We are grateful to all anonymous referees for their useful suggestions.

This work was partially supported by a grant from the National Centre for Research and Development (Strategmed 1/233624/4/NCBR/2014).

\section{Conflict of interest}

The authors declare no conflict of interest.

\section{References}

1. Tímár J, Vizkeleti L, Doma V, et al. Genetic progression of malignant melanoma. Cancer Metastasis Rev 2016; 35: 93 107.

2. Mort RL, Jackson IJ, Patton EE. The melanocyte lineage in development and disease. Development 2015; 142: 620-32.

3. Kulesa PM, Morrison JA, Bailey CM. The neural crest and cancer: a developmental spin on melanoma. Cells Tissues Organs 2013; 198: 12-21.

4. Mull AN, Zolekar A, Wang YC. Understanding melanocyte stem cells for disease modeling and regenerative medicine applications. Int J Mol Sci 2015; 16: 30458-69.

5. Shain AH, Bastian BC. From melanocytes to melanomas. Nat Rev Cancer 2016; 16: 345-58.

6. Roesch A, Paschen A, Landsberg J, et al. Phenotypic tumour cell plasticity as a resistance mechanism and therapeutic target in melanoma. Eur J Cancer 2016; 59: 109-12.
7. Magee JA, Piskounova E, Morrison SJ. Cancer stem cells: impact, heterogeneity, and uncertainty. Cancer Cell 2012; 21: 283-96.

8. Rajabi P, Neshat A, Mokhtari M, et al. The role of VEGF in melanoma progression. J Res Med Sci 2012; 17: 534-9.

9. Li YL, Zhao H, Ren XB. Relationship of VEGF/VEGFR with immune and cancer cells: staggering or forward? Cancer Biol Med 2016; 13: 206-14.

10. Adamcic U, Skowronski K, Peters C, et al. The effect of bevacizumab on human malignant melanoma cells with functional VEGF/VEGFR2 autocrine and intracrine signaling loops. Neoplasia 2012; 14: 612-23.

11. Molhoek KR, Erdag G, Rasamny JK, et al. VEGFR-2 expression in human melanoma: revised assessment. Int J Cancer 2011; 129: 2807-15.

12. Winder M, Virós A. Mechanisms of drug resistance in melanoma. Handb Exp Pharmacol 2018; 249: 91-108.

13. Friedman R. Drug resistance in cancer: molecular evolution and compensatory proliferation. Oncotarget 2016; 7: 11746-55.

14. Manzano JL, Layos L, Bugés C, et al. Resistant mechanisms to BRAF inhibitors in melanoma. Ann Transl Med 2016; 4: 237.

15. Amaral T, Sinnberg T, Meier F, et al. MAPK pathway in melanoma part II-secondary and adaptive resistance mechanisms to BRAF inhibition. Eur J Cancer 2017; 73: 93-101.

16. Sun Y. Tumor microenvironment and cancer therapy resistance. Cancer Lett 2016; 380: 205-15.

17. Bussard KM, Mutkus L, Stumpf K, et al. Tumor-associated stromal cells as key contributors to the tumor microenvironment. Breast Cancer Res 2016; 18: 84.

18. Brandner JM, Haass NK. Melanoma's connections to the tumour microenvironment. Pathology 2013; 45: 443-52.

19. Plaks V, Kong N, Werb Z. The cancer stem cell niche: how essential is the niche in regulating stemness of tumor cells? Cell Stem Cell 2015; 16: 225-38.

20. Nguyen N, Couts KL, Luo Y, et al. Understanding melanoma stem cells. Melanoma Manag 2015; 2: 179-88.

21. Collet G, Skrzypek K, Grillon C, et al. Hypoxia control to normalize pathologic angiogenesis: potential role for endothelial precursor cells and miRNAs regulation. Vascul Pharmacol 2012; 56: 252-61.

22. Borovski T, De Sousa E, Melo F, et al. Cancer stem cell niche: the place to be. Cancer Res 2011; 71: 634-9.

23. Gorgun C, Ozturk S, Gokalp S, et al. Synergistic role of three dimensional niche and hypoxia on conservation of cancer stem cell phenotype. Int J Biol Macromol 2016; 90: 20-6.

24. Leung DW, Cachianes G, Kuang WJ, et al. Vascular endothelial growth factor is a secreted angiogenic mitogen. Science 1989; 246: 1306-9.

25. Senger DR, Connolly DT, Van de Water L, et al. Purification and NH2-terminal amino acid sequence of guinea pig tumor-secreted vascular permeability factor. Cancer Res 1990; 50: 1774-8.

26. Ferrara N, Davis-Smyth T. The biology of vascular endothelial growth factor. Endocr Rev 1997; 18: 4-25.

27. Koch S, Claesson-Welsh L. Signal transduction by vascular endothelial growth factor receptors. Cold Spring Harb Perspect Med 2012; 2: a006502.

28. Goel HL, Mercurio AM. VEGF targets the tumour cell. Nat Rev Cancer 2013; 13: 871-82.

29. Jour G, Ivan D, Aung PP. Angiogenesis in melanoma: an update with a focus on current targeted therapies. J Clin Pathol 2016; 69: 472-83. 
30. Senger DR. Vascular endothelial growth factor: much more than an angiogenesis factor. Mol Biol Cell 2010; 21: 377-9.

31. Kowanetz M, Ferrara N. Vascular endothelial growth factor signaling pathways: therapeutic perspective. Clin Cancer Res 2006; 12: 5018-22.

32. Shibuya M. Structure and function of VEGF/VEGF-receptor system involved in angiogenesis. Cell Struct Funct 2001; 26: 25-35.

33. Hornig C, Barleon B, Ahmad S, et al. Release and complex formation of soluble VEGFR-1 from endothelial cells and biological fluids. Lab Invest 2000; 80: 443-54.

34. Mehnert JM, McCarthy MM, Jilaveanu L, et al. Quantitative expression of VEGF, VEGF-R1, VEGF-R2, and VEGF-R3 in melanoma tissue microarrays. Hum Pathol 2010; 41: 375-84.

35. Kurenova E, Ucar D, Liao J, et al. A FAK scaffold inhibitor disrupts FAK and VEGFR-3 signaling and blocks melanoma growth by targeting both tumor and endothelial cells. Cell Cycle 2014; 13: 2542-53.

36. Prud'homme GJ, Glinka Y. Neuropilins are multifunctional coreceptors involved in tumor initiation, growth, metastasis and immunity. Oncotarget 2012; 3: 921-39.

37. Parker MW, Guo HF, Li X, et al. Function of members of the neuropilin family as essential pleiotropic cell surface receptors. Biochemistry 2012; 51: 9437-46.

38. Grandclement C, Borg C. Neuropilins: a new target for cancer therapy. Cancers (Basel) 2011; 3: 1899-928.

39. Ruffini F, D'Atri S, Lacal PM. Neuropilin-1 expression promotes invasiveness of melanoma cells through vascular endothelial growth factor receptor-2-dependent and -independent mechanisms. Int J Oncol 2013; 43: 297-306.

40. Rushing EC, Stine MJ, Hahn SJ, et al. Neuropilin-2: a novel biomarker for malignant melanoma? Hum Pathol 2012; 43: 381-9.

41. Moriarty WF, Kim E, Gerber SA, et al. Neuropilin-2 promotes melanoma growth and progression in vivo. Melanoma Res 2016; 26: 321-8.

42. Beck B, Driessens G, Goossens S, et al. A vascular niche and a VEGF-Nrp1 loop regulate the initiation and stemness of skin tumours. Nature 2011; 478: 399-403.

43. Streit M, Detmar M. Angiogenesis, lymphangiogenesis, and melanoma metastasis. Oncogene 2003; 22: 3172-9.

44. Wang T, Xiao M, Ge Y, et al. BRAF inhibition stimulates melanoma-associated macrophages to drive tumor growth. Clin Cancer Res 2015; 21: 1652-64.

45. Zhou L, Yang K, Andl T, et al. Perspective of targeting cancer-associated fibroblasts in melanoma. J Cancer 2015; 6: 717-26.

46. Corrie PG, Basu B, Zaki KA. Targeting angiogenesis in melanoma: prospects for the future. Ther Adv Med Oncol 2010; 2: 367-80.

47. Meierjohann S. Hypoxia-independent drivers of melanoma angiogenesis. Front Oncol 2015; 5: 102.

48. Lee N, Barthel SR, Schatton T. Melanoma stem cells and metastasis: mimicking hematopoietic cell trafficking? Lab Invest 2014; 94: 13-30.

49. Hendrix MJ, Seftor EA, Seftor RE, et al. Tumor cell vascular mimicry: novel targeting opportunity in melanoma. Pharmacol Ther 2016; 159: 83-92.

50. Sun B, Zhang D, Zhao N, et al. Epithelial-to-endothelial transition and cancer stem cells: two cornerstones of vasculogenic mimicry in malignant tumors. Oncotarget 2017; 8: 30502-10

51. Fan YL, Zheng M, Tang YL, et al. A new perspective of vasculogenic mimicry: EMT and cancer stem cells (Review). Oncol Lett 2013; 6: 1174-80.
52. Ott PA, Hodi FS, Buchbinder El. Inhibition of immune checkpoints and vascular endothelial growth factor as combination therapy for metastatic melanoma: an overview of rationale, preclinical evidence, and initial clinical data. Front Oncol 2015; 5: 202.

53. Cree IA, Charlton P. Molecular chess? Hallmarks of anticancer drug resistance. BMC Cancer 2017; 17: 10.

54. Tentori L, Lacal PM, Graziani G. Challenging resistance mechanisms to therapies for metastatic melanoma. Trends Pharmacol Sci 2013; 34: 656-66.

55. Wilkens S. Structure and mechanism of ABC transporters. F1000Prime Rep 2015; 7: 14.

56. Housman G, Byler S, Heerboth S, et al. Drug resistance in cancer: an overview. Cancers (Basel) 2014; 6: 1769-92.

57. Wu CP, Ambudkar S. The pharmacological impact of ATPbinding cassette drug transporters on vemurafenib-based therapy. Acta Pharm Sin B 2014; 4: 105-11.

58. Fukunaga-Kalabis M, Herlyn M. Beyond ABC: another mechanism of drug resistance in melanoma side population. J Invest Dermatol 2012; 132: 2317-9.

59. Akiyama K, Ohga N, Hida Y, et al. Tumor endothelial cells acquire drug resistance by MDR1 up-regulation via VEGF signaling in tumor microenvironment. Am J Pathol 2012; 180: 1283-93.

60. Kumar D, Gorain M, Kundu G, et al. Therapeutic implications of cellular and molecular biology of cancer stem cells in melanoma. Mol Cancer 2017; 16: 7.

61. Wilson BJ, Saab KR, Ma J, et al. ABCB5 maintains melanoma-initiating cells through a pro-inflammatory cytokine signaling circuit. Cancer Res 2014; 74: 4196-207.

62. Chartrain M, Riond J, Stennevin A, et al. Melanoma chemotherapy leads to the selection of ABCB5-expressing cells. PLoS One 2012; 7: e36762.

63. Frank NY, Schatton T, Kim S, et al. VEGFR-1 expressed by malignant melanoma-initiating cells is required for tumor growth. Cancer Res 2011; 71: 1474-85.

64. Pappalardo F, Russo G, Candido S, et al. Computational modeling of PI3K/AKT and MAPK signaling pathways in melanoma cancer. PLoS One 2016; 11: e0152104.

65. Aksamitiene E, Kiyatkin A, Kholodenko BN. Cross-talk between mitogenic Ras/MAPK and survival PI3K/AKt pathways: a fine balance. Biochem Soc Trans 2012; 40: 139-46.

66. Wu S, Singh RK. Resistance to chemotherapy and molecularly targeted therapies: rationale for combination therapy in malignant melanoma. Curr Mol Med 2011; 11: 553-63.

67. Strickland LR, Pal HC, Elmets CA, et al. Targeting drivers of melanoma with synthetic small molecules and phytochemicals. Cancer Lett 2015; 359: 20-35.

68. Smalley KS, Haass NK, Brafford PA, et al. Multiple signaling pathways must be targeted to overcome drug resistance in cell lines derived from melanoma metastases. Mol Cancer Ther 2006; 5: 1136-44.

69. Shannan B, Chen Q, Watters A, et al. Enhancing the evaluation of PI3K inhibitors through 3D melanoma models. Pigment Cell Melanoma Res 2016; 29: 317-28.

70. Domigan CK, Ziyad S, Iruela-Arispe ML. Canonical and noncanonical vascular endothelial growth factor pathways: new developments in biology and signal transduction. Arterioscler Thromb Vasc Biol 2015; 35: 30-9.

71. Ni CS, Sun BC, Dong XY, et al. Promoting melanoma growth and metastasis by enhancing VEGF expression. Contemp Oncol (Pozn) 2012; 16: 526-31.

72. Graziani G, Lacal PM. Neuropilin-1 as therapeutic target for malignant melanoma. Front Oncol 2015; 5: 125. 
73. Beazley-Long N, Gaston K, Harper SJ, et al. Novel mechanisms of resistance to vemurafenib in melanoma - V600E $B$-Raf reversion and switching VEGF-A splice isoform expression. Am J Cancer Res 2014; 5: 433-41.

74. Mohammad RM, Muqbil I, Lowe L, et al. Broad targeting of resistance to apoptosis in cancer. Semin Cancer Biol 2015; 35 Suppl: S78-103.

75. Hartman ML, Czyz M. Anti-apoptotic proteins on guard of melanoma cell survival. Cancer Lett 2013; 331: 24-34.

76. Grazia G, Penna I, Perotti V, et al. Towards combinatorial targeted therapy in melanoma: from pre-clinical evidence to clinical application (review). Int J Oncol 2014; 45: 929-49.

77. Gallagher SJ, Tiffen JC, Hersey P. Histone modifications, modifiers and readers in melanoma resistance to targeted and immune therapy. Cancers (Basel) 2015; 7: 1959-82.

78. Berger A, Quast SA, Plötz M, et al. Sensitization of melanoma cells for TRAIL-induced apoptosis by BMS-345541 correlates with altered phosphorylation and activation of Bax. Cell Death Dis 2013; 4: e477.

79. Ichim G, Tait SW. A fate worse than death: apoptosis as an oncogenic process. Nat Rev Cancer 2016; 16: 539-48.

80. Berger A, Quast SA, Plötz M, et al. RAF inhibition overcomes resistance to TRAIL-induced apoptosis in melanoma cells. J Invest Dermatol 2014; 134: 430-40.

81. Delbridge AR, Strasser A. The BCL-2 protein family, BH3mimetics and cancer therapy. Cell Death Differ 2015; 22: 1071-80.

82. Iervolino A, Trisciuoglio D, Ribatti D, et al. Bcl-2 overexpression in human melanoma cells increases angiogenesis through VEGF mRNA stabilization and HIF-1-mediated transcriptional activity. FASEB J 2002; 16: 1453-5.

83. Trisciuoglio D, lervolino A, Zupi G, et al. Involvement of PI3K and MAPK signaling in bcl-2-induced vascular endothelial growth factor expression in melanoma cells. Mol Biol Cell 2005; 16: 4153-62.

84. Trisciuoglio D, Gabellini C, Desideri M, et al. Bcl-2 regulates HIF-1alpha protein stabilization in hypoxic melanoma cells via the molecular chaperone HSP90. PLoS One 2010; 5: e11772.

85. Giorgini S, Trisciuoglio D, Gabellini C, et al. Modulation of bcl-xL in tumor cells regulates angiogenesis through CXCL8 expression. Mol Cancer Res 2007; 5: 761-71.

86. Karl E, Zhang Z, Dong Z, et al. Unidirectional crosstalk between $\mathrm{Bcl}-\mathrm{xL}$ and $\mathrm{Bcl}-2$ enhances the angiogenic phenotype of endothelial cells. Cell Death Differ 2007; 14 1657-66.

87. Zeitlin BD, Zeitlin IJ, Nör JE. Expanding circle of inhibition: small-molecule inhibitors of Bcl-2 as anticancer cell and antiangiogenic agents. J Clin Oncol 2008; 26: 4180-8.

88. Zeitlin BD, Nör JE. Small-molecule inhibitors reveal a new function for $\mathrm{Bcl}-2$ as a proangiogenic signaling molecule. Curr Top Microbiol Immunol 2011; 348: 115-37.

89. Yan B, Kong M, Chen S, et al. VEGF stimulation enhances Livin protein synthesis through mTOR signaling. J Cell Biochem 2010; 111: 1114-24.

90. Sanhueza C, Wehinger S, Castillo Bennett J, et al. The twisted survivin connection to angiogenesis. Mol Cancer 2015; 14: 198

91. Olson OC, Joyce JA. Microenvironment-mediated resistance to anticancer therapies. Cell Res 2013; 23: 179-81.

92. Shimabukuro-Vornhagen A, Draube A, Liebig TM, et al. The immunosuppressive factors IL-10, TGF-beta, and VEGF do not affect the antigen-presenting function of CD40-activated B cells. J Exp Clin Cancer Res 2012; 31: 47.
93. Jones VS, Huang RY, Chen LP, et al. Cytokines in cancer drug resistance: cues to new therapeutic strategies. Biochim Biophys Acta 2016; 1865: 255-65.

94. Voron T, Marcheteau E, Pernot S, et al. Control of the immune response by pro-angiogenic factors. Front Oncol 2014; 4: 70.

95. Peng W, Chen JQ, Liu C, et al. Loss of PTEN promotes resistance to T cell-mediated immunotherapy. Cancer Discov 2016; 6: 202-16.

96. Speiser DE, Ho PC, Verdeil G. Regulatory circuits of T cell function in cancer. Nat Rev Immunol 2016; 16: 599-611.

97. Shrimali RK, Yu Z, Theoret MR, et al. Antiangiogenic agents can increase lymphocyte infiltration into tumor and enhance the effectiveness of adoptive immunotherapy of cancer. Cancer Res 2010; 70: 171-80.

98. Hargadon KM. The extent to which melanoma alters tissue-resident dendritic cell function correlates with tumorigenicity. Oncoimmunology 2015; 5: e1069462.

99. Agostino NM, Saraceni C, Kincaid H, et al. A prospective evaluation of the role of vascular endothelial growth factor (VEGF) and the immune system in stage III/IV melanoma. Springerplus 2015; 4: 186.

100. Ilieva KM, Correa I, Josephs DH, et al. Effects of BRAF mutations and BRAF inhibition on immune responses to melanoma. Mol Cancer Ther 2014; 13: 2769-83.

101. Eljaszewicz A, Wiese M, Helmin-Basa A, et al. Collaborating with the enemy: function of macrophages in the development of neoplastic disease. Mediators Inflamm 2013; 2013: 831387.

102. Kerbel RS. Targeting the VEGF/VEGFR pathway: strategies for improving therapeutic outcomes. In: ASCO Daily News. 2016. https://am.asco.org/daily-news/targeting-vegfvegfrpathway-strategies-improving-therapeutic-outcomes. Accessed 26 May 2016.

103. Donovan P, Patel J, Dight J, et al. Endovascular progenitors infiltrate melanomas and differentiate towards a variety of vascular beds promoting tumor metastasis. Nat Commun 2019; 10: 18.

104. Felcht M, Thomas M. Angiogenesis in malignant melanoma. J Dtsch Dermatol Ges 2015; 13: 125-36.

105. ClinicalTrials.gov. A service of the U.S. National Institutes of Health. 2018. https://clinicaltrials.gov/

106. Corrie PG, Marshall A, Nathan PD, et al. Adjuvant bevacizumab for melanoma patients at high risk of recurrence: survival analysis of the AVAST-M trial. Ann Oncol 2018; 29: 1843-52. 